\title{
The evolving field of bone imaging in multiple myeloma: is it time to abandon skeletal surveys?
}

\author{
H.S. Mian MD MSc* and A. McCurdy $\mathrm{MD}^{\dagger}$
}

Multiple myeloma (MM) is an incurable plasma cell neoplasm that is caused by the clonal proliferation of malignant plasma cells within the bone marrow. In Canada, MM is the 2nd most common hematologic malignancy, with approximately 2800 new cases reported annually ${ }^{1}$. Skeletal events including bone pain and fractures are a major cause of morbidity in MM. At diagnosis, bone pain is present in approximately $60 \%$ of patients ${ }^{2}$. Historically, bony disease in MM has been assessed by skeletal survey (whole-body radiography), which is abnormal in nearly $80 \%$ of patients at the time of diagnosis ${ }^{2}$.

Increasingly, the published literature has supported the under-detection of lytic bone disease by at least $20 \%-25 \%$ on conventional skeletal surveys as compared with advanced imaging (low-dose whole-body computed tomography, magnetic resonance imaging, positron-emission tomography) ${ }^{3,4}$. Guideline bodies, including the International Myeloma Working Group, have recently endorsed the use of advanced imaging at the time of myeloma diagnosis and, in fact, have recommended against conventional skeletal surveys ${ }^{5}$. Furthermore, if any follow-up diagnostic imaging is required, the group recommends use of the same modality as the one used at the time of initial diagnosis. That major shift in the evolving field of bone imaging in MM in a resource-conscious landscape is forcing us to evaluate whether it is time to abandon skeletal surveys altogether.

What is the evidence for the end of the skeletal survey era? There is no doubt that advanced imaging leads to increased detection of lytic disease (and likely more incidental findings as well). That increased detection of lytic disease is of particular importance in cases of highrisk monoclonal gammopathy of unknown significance, smouldering myeloma, or solitary plasmacytoma in which the detection of additional bony disease could in fact lead to a change in treatment and, ultimately, outcome. In a small subset of MM cases in which clinical concern arises or skeletal surveys are negative, advanced imaging might also be helpful in further clarifying particular clinical or radiologic findings. However, for the 2800-plus patients diagnosed with myeloma annually in Canada, many of whom will have abnormal skeletal surveys at baseline, what additional information will advanced imaging provide?
The current answer is that we're not quite sure, and despite the robust evidence for the increased detection of lytic bone disease with advanced imaging in $\mathrm{MM}$, there is a paucity of data showing actual change in clinical management, resource utilization, and patient outcomes with advanced imaging. It is possible that advanced imaging at baseline might in fact lead to overall lower rates of the subsequent and sequential imaging that are sometimes required to clarify or obtain more information after an abnormal skeletal survey. However, at the same time, we will be faced with balancing an increased rate of unanticipated incidental findings that would require further evaluation. Additionally, the cost-benefit advantage of advanced imaging in an already constrained health care system in which a skeletal survey might cost $\$ 50$ and magnetic resonance imaging $\$ 500$ has to be carefully evaluated before any routine implementation.

So where does that leave us in Canada? We recently conducted an internal survey of investigators from the Myeloma Canada Research Network, which revealed significant heterogeneity across the nation. Some clinicians have already abandoned the archaic skeletal survey and aligned their practices with recent international guidelines, stating that understanding the full spectrum of lytic disease in a patient is important. In contrast, other clinicians across the nation continue to hold dearly to skeletal surveys, mentioning reasons such as ease of access and a general skepticism about how the additional information found on advanced imaging would change the clinical treatment plan.

Although that heterogeneity and nonconformity with international guidelines might be uncomforting, it also represents a unique opportunity for Canada to take the lead in this area. As the rest of the world moves ahead with advanced imaging, we might in fact be best suited to study conventional compared with advanced imaging in both retrospective and prospective studies. Additionally, working in a resource-conscious environment might work to our advantage, forcing us to answer questions about the cost-benefit implications of both the increased detection of lytic disease and the unintended consequences of potentially increased rates of incidental findings with advanced imaging. 
Before we discard the skeletal survey, let's not only re-evaluate the existing evidence, but also, through collaborative efforts with the Myeloma Canada Research Network, add to the existing evidence base and position ourselves to lead the discussion about it.

\section{CONFLICT OF INTEREST DISCLOSURES}

We have read and understood Current Oncology's policy on disclosing conflicts of interest, and we declare that we have none.

\section{AUTHOR AFFILIATIONS}

*Department of Oncology, Juravinski Cancer Centre, McMaster University, Hamilton, and 'Department of Hematology, The Ottawa Hospital, Ottawa, ON.

\section{REFERENCES}

1. Myeloma Canada. Incidence and Prevalence in Canada [Web page]. Dorval, QC: Myeloma Canada; n.d. [Available at: https://www.myelomacanada.ca/en/about-multiple-myeloma/ what-is-myeloma/incidence-and-prevalence-in-canada; cited 18 March 2018]

2. Kyle RA, Gertz MA, Witzig TE, et al. Review of 1027 patients with newly diagnosed multiple myeloma. Mayo Clin Proc 2003;78:21-33.

3. Regelink JC, Minnema MC, Terpos E, et al. Comparison of modern and conventional imaging techniques in establishing multiple myeloma-related bone disease: a systematic review. Br J Haematol 2013;162:50-61.

4. Wolf MB, Murray F, Kilk K, et al. Sensitivity of whole-body СT and MRI versus projection radiography in the detection of osteolyses in patients with monoclonal plasma cell disease. Eur J Radiol 2014;83:1222-30.

5. Hillengass J, Usmani S, Rajkumar SV, et al. International Myeloma Working Group consensus recommendations on imaging in monoclonal plasma cell disorders. Lancet Oncol 2019;20:e302-12. 